\title{
Modelación de indicadores para el control de la dilución interna en yacimientos cubanos de níquel y cobalto
}

\section{Modeling of indicators for controlling internal dilution in Cuban nickel and cobalt deposits}

Lázaro Francisco Fernández-Martínez ${ }^{1 *}$, Ramón Eddie Peña-Abreu ${ }^{1}$, Alfonso Puentes-Martin²

\footnotetext{
${ }^{1}$ Empresa productora de níquel y cobalto Ernesto Che Guevara, Moa, Holguín, Cuba

${ }^{2}$ Centro Universitario de Banes, Holguín.
}

*Autor para la correspondencia: Ifernandez@ecg.moa.minem.cu

\begin{abstract}
Resumen
Las intercalaciones de material amenífero dentro del horizonte menífero de los yacimientos ferroniquelíferos son la causa principal de la dilución interna, la cual ocasiona una baja eficiencia industrial. Por lo difícil que resulta pronosticar estas intercalaciones en puntos sin datos dentro de las redes de muestreo, el propósito de esta investigación fue estimar la distribución probabilística espacial de estas. Para ello se empleó el modelo markoviano de yacimientos a fin de crear índices de complejidad que cuantifiquen la frecuencia de aparición y la dispersión de las intercalaciones. Estos índices permiten establecer los sectores con mayor probabilidad de ocurrencia de la dilución interna, lo que es de gran utilidad en la planificación minera.
\end{abstract}

Palabras clave: intercalaciones ameníferas; dilución interna; selectividad minera; indicadores de la complejidad; modelación markoviana.

\section{Abstract}

The intercalations of ameniferous material within the meniferous horizon of the ferronickel deposits are the main cause of the internal dilution, which causes a low industrial efficiency. The purpose of this study is estimating the spatial probabilistic distribution of intercalations. It is very difficult 
predicting these intercalations in points without data in the sampling networks. To fulfill this purpose the Markovian model of deposits was used in order to create complexity indexes that quantify the frequency of appearance and the dispersion of the intercalations; these results allowed establishing the areas with the highest probability of occurring internal dilution, what is very useful in mining planning.

Keywords: ameniferous intercalations; internal dilution; mining selectivity; indicators of complexity; Markovian modeling.

\section{INTRODUCCIÓN}

La dilución interna es el fenómeno que se produce durante la extracción de las menas cuando estas se mezclan con intercalaciones ameníferas (IA), ocasionando un deterioro de la calidad del mineral que se abastece al proceso industrial.

La gran variabilidad y complejidad geológicas de las cortezas de meteorización ferroniquelíferas del macizo ofiolítico Moa-Baracoa es la causa de la aparición de las IA, cuyos bajos contenidos de $\mathrm{Ni}$ y Co y altos de elementos nocivos al proceso fabril redundan en ineficiencia industrial.

Por sus propiedades físicas, reducidas dimensiones y su carácter discreto y disperso, las IA no siempre son detectadas durante la exploración y su pronóstico con métodos de interpolación no ha tenido éxito, por lo que este estudio se propuso desarrollar un modelo de la distribución probabilística espacial de las IA, sustentado en los modelos markovianos, para establecer índices de esta complejidad en los yacimientos lateríticos cubanos para el manejo de la dilución interna.

Los modelos markovianos se fundamentan en la clasificación multivariada de los valores geoquímicos obtenidos en la exploración (Peña-Abreu 2015). Las clases establecidas constituyen sus variables y cada clase explica un comportamiento geoquímico que se enmarca en los rangos de las variables analizadas en la exploración. El conjunto de todas las clases cubre el comportamiento geoquímico del yacimiento, por lo que contiene materiales meníferos y ameníferos separados en dos subconjuntos de clases mutuamente excluyentes. 
A partir de este modelo es posible crear índices que cuantifiquen la frecuencia de aparición y la dispersión de las IA. La particularidad de dicho modelo es que permite, a través de la expresión de su frecuencia de aparición y entropía, revelar las medidas de presencia y dispersión de las intercalaciones.

\section{MATERIALES Y MÉTODOS}

La investigación tuvo lugar en los yacimientos Punta Gorda, Yagrumaje Norte, Yagrumaje Sur y Camarioca Este (Base Minera de la empresa Che Guevara-ECG), actualmente en fase de explotación, en los cuales son frecuentes las IA.

Como punto de partida se seleccionaron las variables a emplear, se validaron los datos, se analizaron las regularidades probabilísticas de las variables y posteriormente se realizó la clasificación multivariada que facilitó la modelación probabilística con el modelo de Markov.

La ausencia de normalidad de la distribución probabilística de las variables geoquímicas, registradas en la base de datos de los cuatro yacimientos, que además, no se ajustan a ningún conjunto de las distribuciones conocidas y de las más empleadas (Peña-Abreu y Perdomo 2014 y Tomás 2017), justifica el empleo de modelos probabilísticos no paramétricos en sustitución de los modelos de interpolación para modelar espacialmente las IA.

Mediante la aplicación del modelo markoviano se obtiene la distribución probabilística espacial de los tipos de IA a partir de la cual se diseñan índices que cuantifican la complejidad de las intercalaciones en un determinado sector. Para validar estos índices se seleccionó el bloque 048 del yacimiento Punta Gorda, perforado y muestreado en la red de $8,33 \mathrm{~m}$ de lado, el cual cuenta con una extensa información geológica que permite verificar la efectividad de los índices en el mapeo de la complejidad de las IA, para el manejo de la dilución interna.

\subsection{Selección de las variables y validación de los datos}

Fernández, Rojas-Purón y Belete (2015) develan que las variables geoquímicas son las más representativas para el estudio de las IA y fijan el contenido límite $(\mathrm{CL})$ que determina la condición de mena $(\mathrm{Ni} \geq 0,9 \%)$, por lo que todo el material que incumple el $\mathrm{CL}$ no es 
mena y puede constituir una intercalación amenífera, de ahí que el CL defina la geometría de las IA.

Las bases de datos de los yacimientos referidos abarcan seis variables cuantitativas: $\mathrm{Fe}, \mathrm{Ni}, \mathrm{Co}, \mathrm{SiO} 2, \mathrm{MgO}$ y $\mathrm{Al2O}$; para el yacimiento Punta Gorda, en particular, contienen solo las tres primeras: $\mathrm{Fe}, \mathrm{Ni}$ y $\mathrm{Co}$. Se dispuso de 309790 registros del muestreo de exploración que incluye, para cada intervalo, los elementos químicos, la clasificación litológica y la clasificación menífera.

El Microsoft Excel 2010, el Microsoft Access 2010 y el Lenguaje de Consulta Estructurado (SQL) fueron las herramientas de gestión empleadas para detectar valores duplicados y perdidos, así como falta de sincronización entre las tablas y los valores anómalos en las bases de datos. Esta limpieza evitó cálculos innecesarios en la modelación, la que emplea algoritmos que se encarecen con la cantidad de datos, a la vez que garantiza la calidad descriptiva del modelo de clasificación.

El proceso de análisis para la selección del sistema de variables ya ha sido expuesto en trabajos anteriores (Peña-Abreu y VeraSardiñas 2013), por lo que no se creyó necesario repetirlo.

\subsection{Regularidades probabilísticas de las variables}

El conocimiento de las regularidades probabilísticas de las variables aleatorias eleva la precisión de la modelación y de los resultados que de ella se obtienen. En los yacimientos lateríticos del oriente cubano varios autores aplican la prueba de normalidad a una variable en todo el conjunto de datos (Legrá 1999; Cuador-Gil 2002; VeraSardiñas 2001; Martínez-Vargas 2007). Esto no permite revelar todos sus comportamientos, se obvia la georreferenciación y propicia el empleo de modelos incorrectos. Peña-Abreu y Perdomo (2014) proponen un enfoque direccional en el análisis del comportamiento probabilístico de las variables, que se utilizó por Tomás (2017) para analizar las bases de datos de los cuatro yacimientos mencionados.

Las pruebas de ajuste se aplicaron a todas las variables en cada intervalo del sondeo y en ocho direcciones respecto al centro de masa de la red en cada yacimiento. Los resultados de los trabajos de Peña- 
Abreu y Perdomo (2014) y Tomás (2017) se aceptaron como válidos en esta investigación.

\subsection{Clasificación del comportamiento geoquímico}

El comportamiento geoquímico en los yacimientos lateríticos del oriente cubano ha sido clasificado por Lavaut $(1987,2004)$. Para el control técnico de la producción en la industria se empleó, además, una clasificación basada en dos variables (contenido de $\mathrm{Fe}$ y contenido de $\mathrm{Ni}$ ), como resultado de la cual se consideran mena las clases LB y SB, mientras las otras pueden constituir material de intercalación (Tabla 1).

La clasificación de la variedad de materiales presentes en los yacimientos lateríticos cubanos de $\mathrm{Ni}$ y $\mathrm{Co}$, según sus características geoquímicas, requiere de un enfoque multivariado. En este estudio se empleó la metodología de clasificación propuesta por Peña-Abreu y Vera-Sardiñas (2013), que consiste en combinar el análisis de componentes principales (ACP) con la clasificación por reconocimiento de patrones; ello se aplicó a los cuatro yacimientos de la Base Minera de la ECG. La combinación del ACP y el análisis de clasificación (AC) resultaron eficaces en otras investigaciones para clasificar comportamientos (Peña-Abreu et al. 2007; Durán y Fernández 2009; Hernández, Cobo y Pérez 2010). 
Tabla 1. Clasificación de las lateritas cubanas según horizontes geológicos y tecnológicos en la base minera de la ECG

\section{CLASIFICACIÓN SEGÚN LAVAUT (2003)}

\begin{tabular}{|c|c|c|c|c|c|c|}
\hline Horizontes & Descripción & Código & Horizontes & \multicolumn{2}{|c|}{ Descripción } & Código \\
\hline 1 & $\begin{array}{l}\text { Ocres inestructurales } \\
\text { con perdigones }\end{array}$ & OICP & 4 & \multicolumn{2}{|c|}{$\begin{array}{l}\text { Ocres estructurales } \\
\text { iniciales }\end{array}$} & OEI \\
\hline 2 & Ocres inestructurales & OI & 5 & \multicolumn{2}{|c|}{ Roca madre lixiviada } & RML \\
\hline 3 & $\begin{array}{l}\text { Ocres estructurales } \\
\text { finales }\end{array}$ & OEF & 6 & \multicolumn{2}{|c|}{ Roca madre alterada } & RMA \\
\hline \multicolumn{7}{|c|}{ HORIZONTES TECNOLÓGICOS EN LA BASE MINERA DE LA ECG } \\
\hline Horizontes & \multicolumn{3}{|c|}{ Descripción } & Código & \multicolumn{2}{|c|}{ Límites en \% } \\
\hline \multirow{3}{*}{$\begin{array}{c}\text { Materiales } \\
\text { no } \\
\text { meníferos }\end{array}$} & \multicolumn{3}{|c|}{ Material ferroso fuera de balance } & $\mathrm{FF}$ & $\mathrm{Ni}<0,7$ & $20<\mathrm{Fe}<30$ \\
\hline & \multicolumn{3}{|c|}{ Material ferroso de balance } & FB & $\mathrm{Ni}<0,70$ & $\mathrm{Fe}>=30$ \\
\hline & \multicolumn{3}{|c|}{ Material laterítico niquelífero fuera de balance } & LF & $0,70<\mathrm{Ni}<0,90$ & $\mathrm{Fe}>=35$ \\
\hline \multirow{3}{*}{ Menas } & \multicolumn{3}{|c|}{ Menas lateríticas de balance } & LB & $\mathrm{Ni}>=0,9$ & $\mathrm{Fe}>=35$ \\
\hline & \multicolumn{3}{|c|}{$\begin{array}{l}\text { Mena serpentinítica niquelífera friable de } \\
\text { balance }\end{array}$} & SB & $\mathrm{Ni}>=0,9$ & $12<\mathrm{Fe}<35$ \\
\hline & \multicolumn{3}{|c|}{ Mena serpentinítica dura de balance } & SD & $\mathrm{Ni}>=0,9$ & $\mathrm{Fe}<12$ \\
\hline \multirow[t]{2}{*}{ Roca madre } & \multicolumn{3}{|c|}{$\begin{array}{l}\text { Mena serpentinítica niquelífera fuera de } \\
\text { balance }\end{array}$} & SF & $0,70<\mathrm{Ni}<0,90$ & $12<\mathrm{Fe}<35$ \\
\hline & \multicolumn{3}{|c|}{ Roca estéril } & RE & $\mathrm{Ni}<0,70$ & $\mathrm{Fe}<20$ \\
\hline
\end{tabular}

Del análisis de clasificación se obtienen las variables activas, las cuales constituyen un sistema de variables representativas e independientes que se utilizan en el análisis de clasificación. Las clases obtenidas se denominaron tipos de comportamiento geoquímico (TCG).

\subsection{Modelación probabilística de los yacimientos}

El modelo markoviano de yacimientos propuesto por Peña-Abreu (2014 y 2015) se fundamenta en las probabilidades condicionales de ocurrencia de una clase en un punto, conocida la clase que contiene en el punto inmediato anterior, en una dirección fijada de la red de muestreo del yacimiento. Para una dirección fija se genera la matriz (1) de probabilidades condicionales, la dirección horizontal se denota con $\tau$, la vertical con $\xi$, el intervalo en el perfil del yacimiento con $\mathrm{r}$ y los pasos entre el punto de origen y destino con s (Figura 1 ). 
Para este trabajo solo se tomaron las direcciones verticales $0^{\circ}$ y $180^{\circ}$. A $\pi_{r, s}^{\tau, \xi}$ se le denomina matriz de transferencia de la dirección $\tau, \xi$ en el nivel $r$ a s pasos. El modelo que integra todas las direcciones se expone en (2):

$$
\begin{gathered}
\boldsymbol{\pi}_{r, s}^{\tau, \xi}=\left\{\boldsymbol{p}_{i, j}\right\}_{r, s}^{\tau, \xi}=\left(\begin{array}{ccc}
p_{1,1} & \cdots & p_{1, n} \\
\vdots & \ddots & \vdots \\
p_{n, 1} & \cdots & p_{n, n}
\end{array}\right) \\
\Pi(t)=\left(\begin{array}{ccc}
\boldsymbol{\pi}_{1,1}^{\tau, \xi}(t) & \cdots & \boldsymbol{\pi}_{1, u}^{\tau, \xi}(t) \\
\vdots & \ddots & \vdots \\
\boldsymbol{\pi}_{v, 1}^{\tau, \xi}(t) & \cdots & \boldsymbol{\pi}_{v, u}^{\tau, \xi}(t)
\end{array}\right)
\end{gathered}
$$

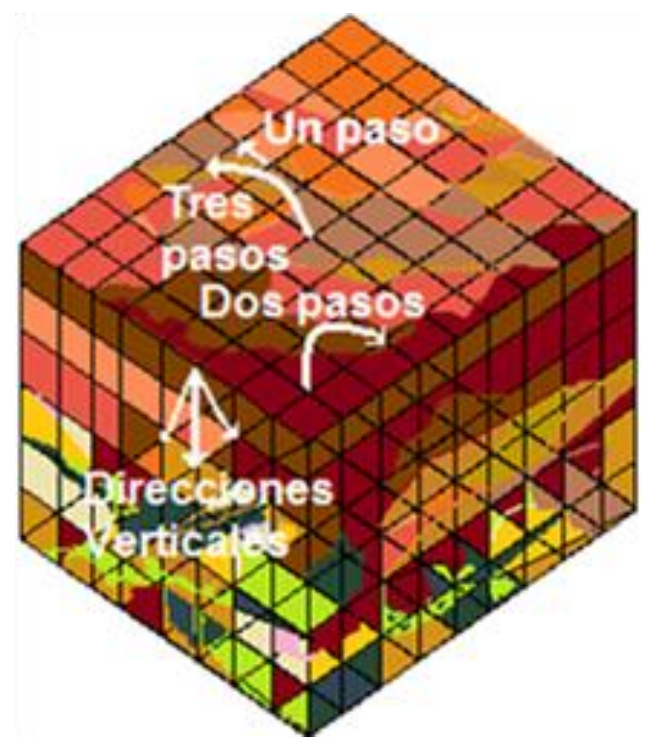

Figura 1. Direcciones y relación entre puntos en la red de muestreo con el modelo markoviano.

Los índices $\mathrm{v}$ y u denotan el orden de la discretización del plano horizontal y vertical, respectivamente. El funcional de georreferenciación de la información se expone en la expresión (3):

$\boldsymbol{g}(\boldsymbol{t}, \boldsymbol{r})=\left(\begin{array}{ccc}\boldsymbol{g}_{1,1, \xi}(t, r(\varphi, \theta, \rho)) & \cdots & g_{1, u, \xi}(t, r(\varphi, \theta, \rho)) \\ \vdots & \ddots & \vdots \\ g_{v, 1, \xi}(t, r(\varphi, \theta, \rho)) & \cdots & g_{v, u, \xi}(t, r(\varphi, \theta, \rho))\end{array}\right)$

El modelo de yacimiento se integra en (4):

$X(t, r)=\widehat{\Pi}(t) \circ g(t, r)$

Como metodología para la interpretación del modelo markoviano se empleó una notación que indexa las clases ameníferas que son factibles de constituir IA. Los resultados de la modelación se exponen 
según estos índices y consisten en distribuciones de probabilidad para cada combinación de las clases entre dos puntos del yacimiento.

La proporción de intercalaciones que se pueden encontrar en una zona del yacimiento dependerá de las distribuciones de probabilidad de las combinaciones en las que aparezcan las clases ameníferas dentro de la zona de menas. Según Duarte (2009), en los modelos markovianos las probabilidades inferiores al $10 \%$ resultan poco significativas, por ello las combinaciones que presenten probabilidades por debajo de ese valor no se tendrán en cuenta.

Se debe delimitar en la zona elegida el techo y el fondo de la zona de menas donde pueden estar contenidas las IA. Se denota con $\varsigma$ el índice del intervalo que indica en un pozo el comienzo de la zona de menas y ı el límite inferior de la zona de menas.

Para estimar con el modelo markoviano de yacimiento el comportamiento de los materiales ameníferos que generan las intercalaciones se analizan solo los índices impares de $\mathrm{j}$ en la expresión (5), lo que se denota:

$\hat{\jmath}=\{j=\overline{1, n} ; j \operatorname{div} 2 \neq 0\}$

Las matrices de transferencias para este caso se exponen en (6):

$\pi_{r, S}^{\tau, \xi}(\boldsymbol{i}, \boldsymbol{j})=\left\{\boldsymbol{p}_{i \hat{j}}\right\}_{i=\overline{1, n}}$

Las distribuciones $\pi_{r, s}^{\tau, \xi}(i, j)$ analizadas en el perfil para todos los pasos s para una dirección horizontal $\tau$, sin movimiento en las direcciones verticales $(\xi=0)$, permite calcular la probabilidad de cada combinación de clases para cada una de las direcciones horizontales en un sector angular $d \varphi$, en la profundidad del perfil, lo que se expresa en (7).

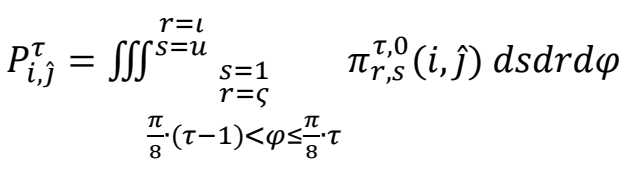

Las probabilidades $\mathrm{P}_{\mathrm{i}, \hat{\mathrm{j}}}^{\tau}$ que no superen el $10 \%$ se excluyen. En cada dirección $\tau$ existen $\frac{\mathrm{n}^{2}}{2}$ combinaciones posibles de clases que deben ser analizadas, de ellas se elige la combinación más probable por (8): 
$\widehat{P_{l}^{\tau}}=\max _{i, \hat{\jmath}} P_{i, \hat{\jmath}}^{\tau}$

De esta forma, a cada dirección $\tau$ se le asocia la combinación más probable que incluye al menos un material amenífero. Las combinaciones en que aparezcan menas en el origen (índice i) indican que la mena puede ser sucedida de un material amenífero. Si en el origen es material amenífero significa que se extiende la aparición de materiales ameníferos.

\section{METOdología PARA eVAluAR LA COMPLEJidAd DE LAS INTERCALACIONES NO INDUSTRIALES}

La complejidad que generan las IA en el área minera es producto de dos factores: el primero se relaciona con la cantidad de IA que sustituyen a la mena en el horizonte de menas y está representado por el índice de intercalaciones; el segundo está dado por el grado de dispersión que presentan estas, lo cual ofrece la medida de la posibilidad de su separación selectiva durante la minería, como resultado de la entropía del índice de intercalaciones. En una zona se puede calcular el índice de intercalaciones de material amenífero, que debe ser la relación entre los valores significativos de las combinaciones con material amenífero y el total de combinaciones de los tipos materiales en la zona; este último para cada dirección horizontal $\tau$ se expresa en (9):

$$
\begin{aligned}
& P_{i j}^{\tau}=\iiint_{\substack{r=l \\
s=u}}^{\substack{s=1 \\
r=\varsigma}} \quad \pi_{r, s}^{\tau, 0}(i, j) d s d r d \varphi \\
& \frac{\pi}{8} \cdot(\tau-1)<\varphi \leq \frac{\pi}{8} \cdot \tau
\end{aligned}
$$

Entonces el índice en forma integral se escribe en la expresión (10):

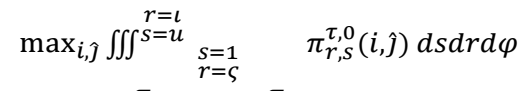

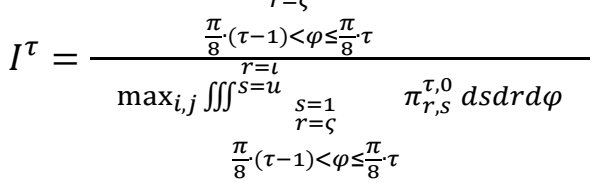

Lo que es equivalente a la expresión reducida (11):

$$
I^{\tau}=\frac{\widehat{P_{l}^{\tau}}}{P_{i j}^{\tau}}
$$


Este índice es la probabilidad de encontrar materiales ameníferos en una dirección de la zona que se estudie y cuanto más cercano sea a la unidad mayor será dicha probabilidad.

Lo anteriormente explicado es la expresión continua del índice (11), sin embargo, se tiene un espacio discretizado en las redes de muestreo y por las clases de comportamiento, las clases pueden comportarse de diferentes formas probabilísticas, por lo que se deben emplear métodos no paramétricos y que aprovechen la discretización del espacio.

La expresión (9) es una matriz de las distribuciones de probabilidad, en forma discreta se debe emplear la distribución binomial para construir las distribuciones, lo cual se expone en (12):

$P_{i}^{\tau}=\max _{j} \sum_{j=1}^{n} \mathcal{B}\left(n_{i, j} ; \mathcal{N} ; \alpha\right)$

Donde $n_{\mathrm{i}, \mathrm{j}}$ es el número de casos en los que $\pi_{\mathrm{r}, \mathrm{s}}^{\tau, \xi}(i, j) \geq 0,1, \xi=0$, r es el nivel de los intervalos en el perfil y s los pasos. $\mathcal{N}$ es el número total de intervalos analizados en la dirección $\tau, \xi=0, r, s$. $\alpha$ es la probabilidad de error que se fija en la distribución binomial, se toma $\alpha=0,05$. Para todas las combinaciones de clases de (12) se obtiene (13):

$P^{\tau}=\max _{i} P_{i}^{\tau}$

De forma similar, se transforma (12) en (14) para las combinaciones que contienen materiales ameníferos:

$\widehat{P^{\tau}}=\max _{i} \widehat{P_{l}^{\tau}}$

De (11), (13) y (14) se modifica el coeficiente en su forma discreta en (15):

$I^{\tau}=\frac{\widehat{P \tau}}{P^{\tau}}$

El índice $I^{\tau}$ expresa la probabilidad de existencia de IA en la dirección $\tau$, y puede expresarse en porcentaje. Cuando $\mathrm{I}^{\tau} \geq 10 \%$ la aparición de IA en la dirección señalada es posible. $I^{\tau}$ expresa la complejidad de las IA y refleja su presencia en relación con el total de materiales en el horizonte menífero. El mapeo de $\mathrm{I}^{\tau}$ indicará las zonas favorables para la existencia de estos materiales. 
El comportamiento entrópico de las IA en el área se calcula mediante la expresión (16) a partir del coeficiente $\mathrm{I}^{\tau I^{\mathrm{T}}}$. El índice $\mathrm{I}^{\mathrm{\tau}} \in[0 ; 1]$, por lo que los valores de $\varepsilon_{\text {IA }}^{\tau}$ pertenecen a un subintervalo de $[0 ; 1][0 ; 1] y$ los menores valores se alcanzan en $I^{\tau}=0 \quad I^{\mathbb{T}}=0$ ó $I^{\tau}=1 I^{\mathbb{\tau}}=1$; mientras que el mayor valor se encuentra en el intervalo $I^{\tau} \in[0,3 ; 0,4]$ (Figura 2), al cual se le denomina crítico y se denota $\omega_{\text {Cri. }}$. Se debe notar que tanto $\mathrm{I}^{\tau} \operatorname{como} \varepsilon_{\mathrm{IA}}^{\tau}$ son magnitudes adimensionales.

$\varepsilon_{I A}^{\tau}=-I^{\tau} \cdot \ln I^{\tau}$

Los valores de $\varepsilon_{\mathrm{IA}}^{\tau}$ en $\omega_{\text {Cri }}$ indican el grado de mayor dispersión de las IA en el área estudiada y permiten elaborar mapas de isolíneas que definen diferentes dominios de complejidad. En los sectores con mayor entropía resulta más complejo reducir la DI.

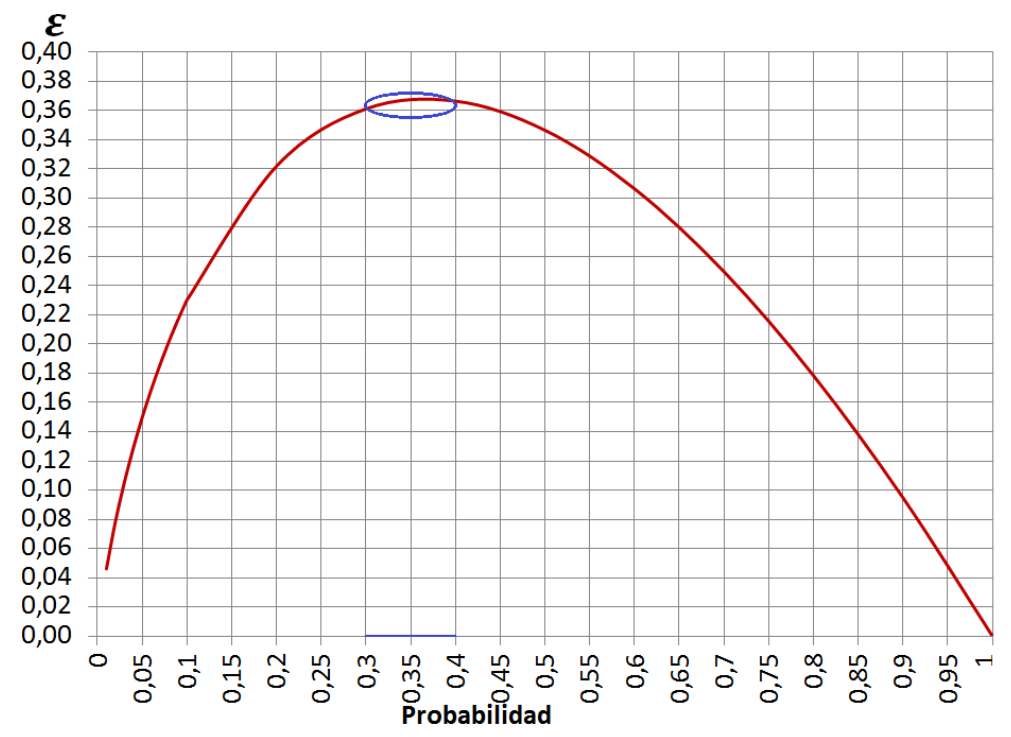

Figura 2. Entropía $\varepsilon_{I A}^{\tau}$ en el intervalo $[0 ; 1]$.

La coincidencia en una zona de los mayores valores de $\mathrm{I}^{\tau}$ y de $\varepsilon_{\text {IA }}^{\tau}$ expresa la máxima complejidad en la minería, por la alta probabilidad de encontrar IA con un grado de dispersión tal, que no será posible extraerlas selectivamente con la tecnología minera existente. En estos sectores el riesgo de dilución interna es máximo.

\section{RESULTADOS Y DISCUSIÓN}

En la limpieza de la base de datos se excluyeron, por escasez de información, el $11 \%$ de las muestras obtenidas en la exploración de 
los cuatro yacimientos y el 2,5\% por anomalías geoquímicas. Los casos excluidos no afectan la integralidad de la base de datos y permiten un comportamiento adecuado del modelo.

EI ACP permitió definir un sistema de variables geoquímicas que describe el comportamiento general del producto residual del intemperismo de los yacimientos de la Base Minera (Figura 3). Este sistema se emplea para la clasificación multivariada.

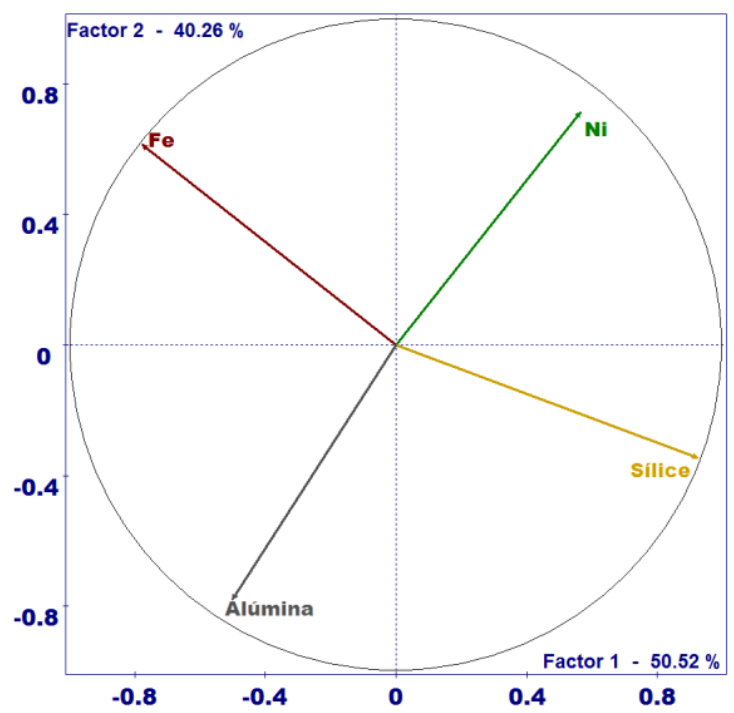

Figura 3. ACP del sistema de variables para la clasificación.

\subsection{Resultados de la clasificación}

En el sistema de las variables $\mathrm{Fe}, \mathrm{SiO} 2, \mathrm{Al} 2 \mathrm{O} 3$ y Ni, el análisis de clasificación realizado con el software Spad 5.1 se muestra en la Figura 4. Se obtuvieron once clases que subdividen los ocho horizontes tecnológicos y cada clase se subdivide en dos, según sean menas o no, por lo que se obtienen 22 tipos diferentes de materiales, once de los cuales son meníferos y los once restantes no lo son, según el $\mathrm{CL}$ establecido. Los materiales no meníferos se identifican por el número de la clase_cero (Ej. 2_0) y los meníferos por el número de la clase_número de la clase (Ej. 2_2). La composición sustancial de las once clases se expone en la Figura 5. 


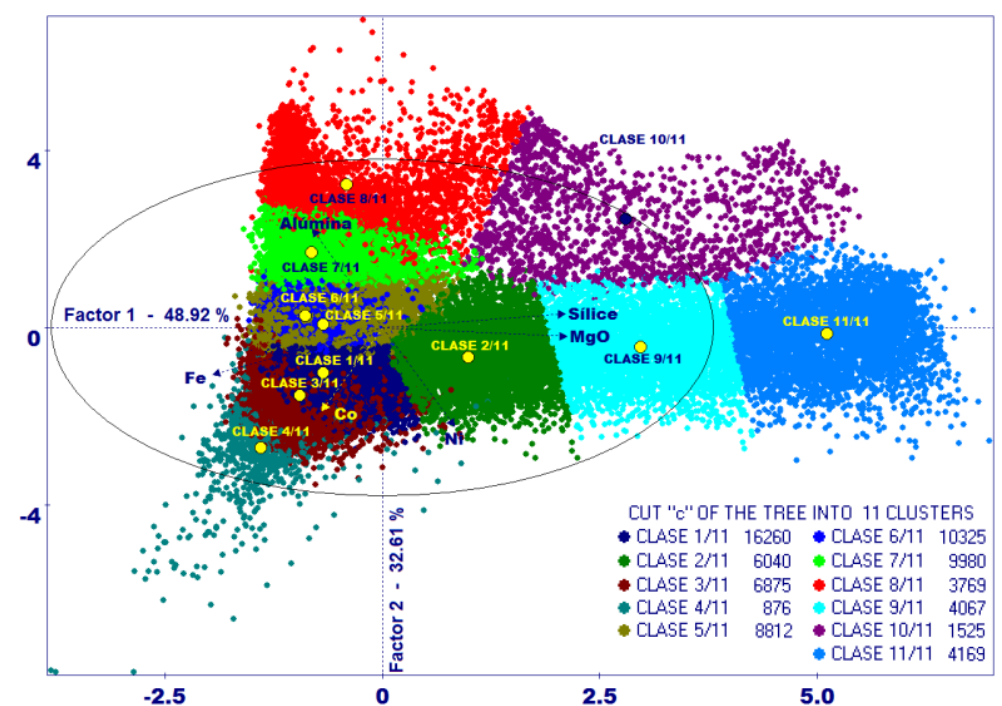

Figura 4. Clasificación en el sistema de variables $\mathrm{Ni}, \mathrm{Fe}, \mathrm{SiO}_{2}$ y $\mathrm{Al}_{2} \mathrm{O}_{3}$.

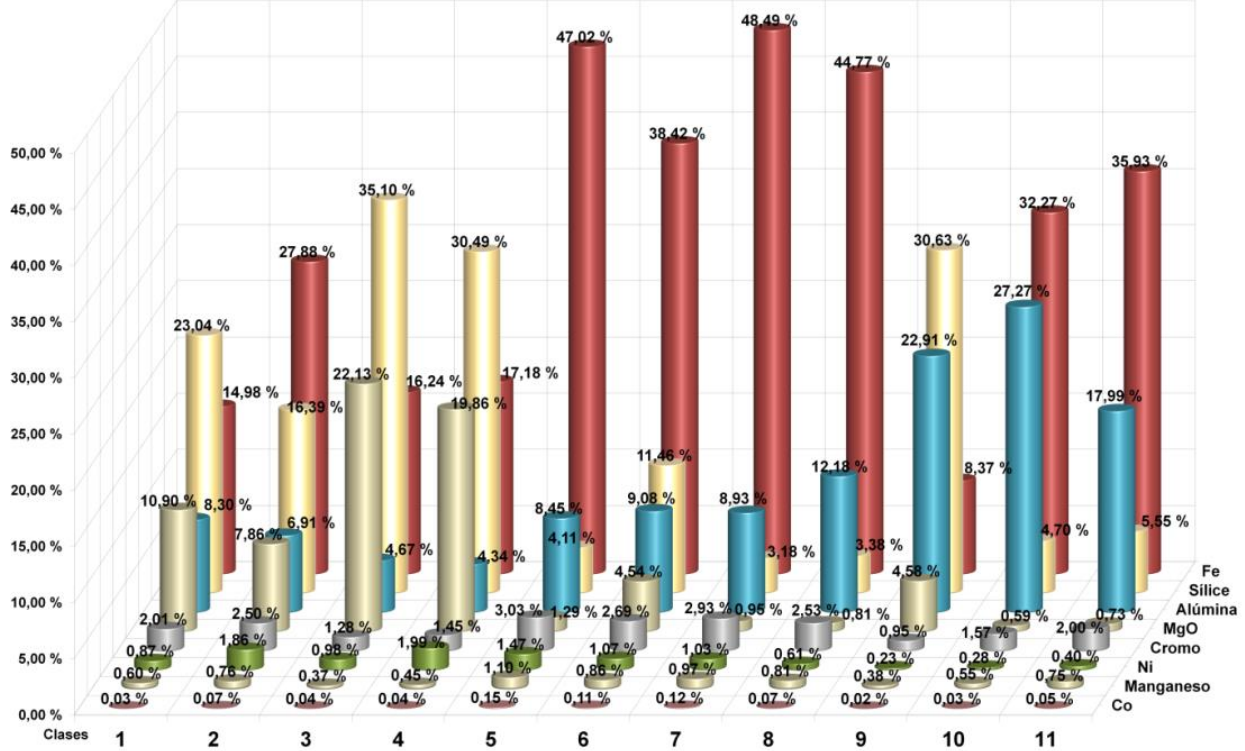

Figura 5. Composición sustancial de las clases.

Se observa que las primeras cuatro clases y la novena están compuestas fundamentalmente de material que no supera el $35 \%$ de Fe. La Figura 6 muestra la subdivisión en menas y no menas, en ella se revela que el $99 \%$ de la clase 2 es mena, mientras las clases $8,9,10$ y 11 tienen un alto porcentaje de material amenífero. La clase 5 se compone exclusivamente de material menífero. Los materiales ameníferos, constituyentes de las intercalaciones, están bien representados en las clases $1,8,910$ y 11 . 


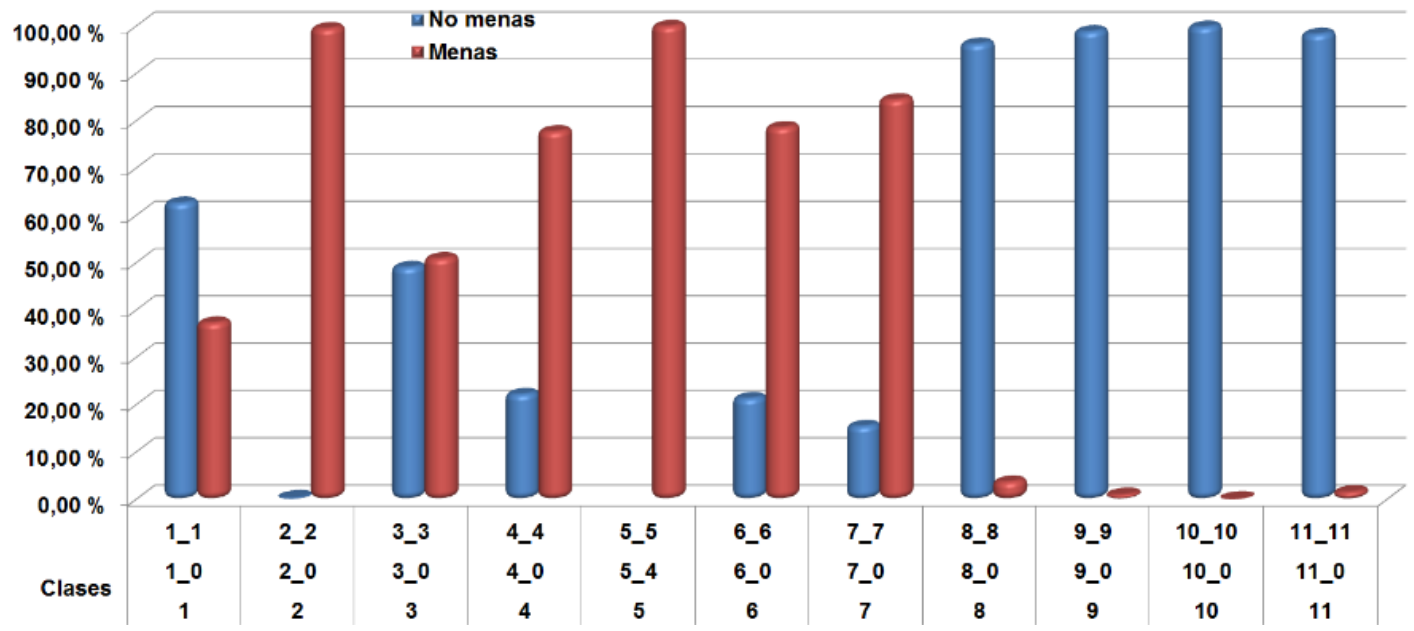

Figura 6. Proporción de menas y no menas en cada clase.

En la Figura 7 aparecen separados los elementos minoritarios (encima) y mayoritarios (debajo). Entre los últimos destaca el contenido de Fe superior a $35 \%$ en las clases de la 5_0 a la 8_0, coincidente con valores bajos de $\mathrm{MgO}$, lo que denota la posición del material amenífero en la parte alta del perfil de meteorización; lo contrario a lo que ocurre en las clases de la 2_0 a la 4_0, donde se vinculan a las partes más bajas del perfil. Entre los minoritarios se destacan los altos valores de $\mathrm{Ni}$ en las clases 2_0 y 4_0, asociados a altos valores de $\mathrm{Mg}$ en esas mismas clases, característico de zonas bajas del perfil de meteorización. Altos valores de alúmina conjuntamente con bajos de Fe y de $\mathrm{Mg}$ en la clase 9_0 sugieren una vinculación de las IA con rocas básicas.

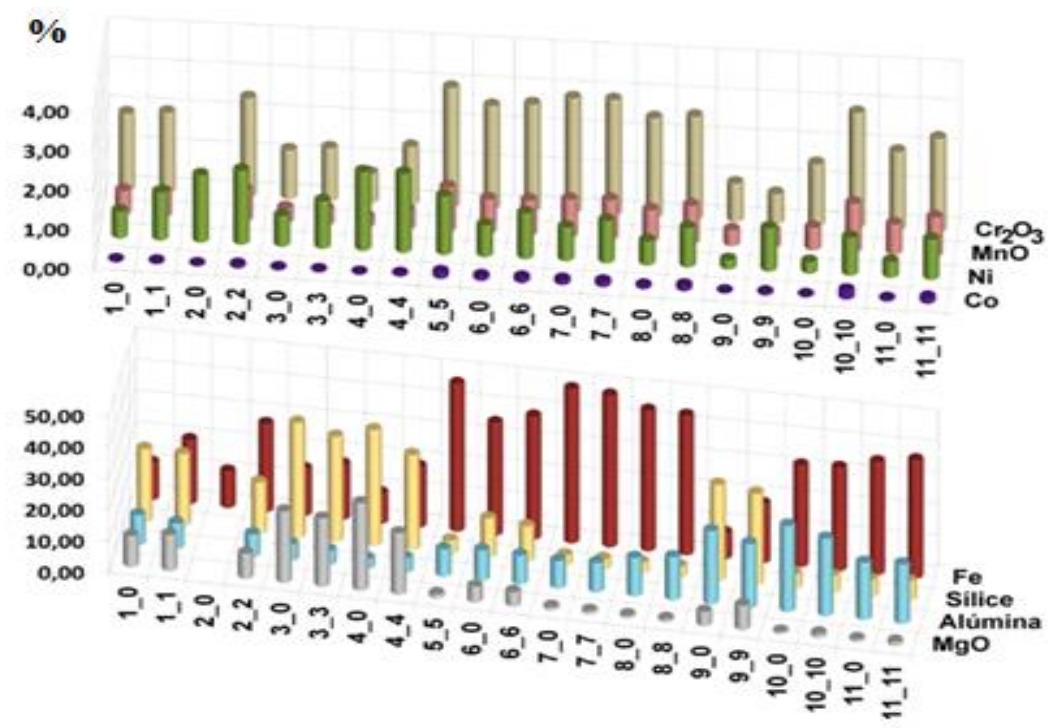

Figura 7. Composición sustancial de las clases subdivididas según CL. 


\subsection{Análisis de complejidad de las intercalaciones no industriales en el bloque 048 de PG}

Los cálculos de $\mathrm{I}^{\tau}$ obtenidos de la estimación entre puntos de la malla cuadrada, de ocho metros de lado en el bloque 0-48 de PG, proporciona la posibilidad de mapear su comportamiento (Figura 8). El riesgo de dilución interna se determina al combinar ambos índices (Figura 9) y se interpreta en mapas de complejidad. A mayores valores de $I^{\tau}$ y menores de $\varepsilon_{\mathrm{IA}}^{\tau}$ crecen las potencialidades de reducción de la DI. Esta combinación se presenta en sectores con abundancia de IA y una tendencia a su concentración espacial o con mayores dimensiones, lo cual favorece su extracción selectiva.
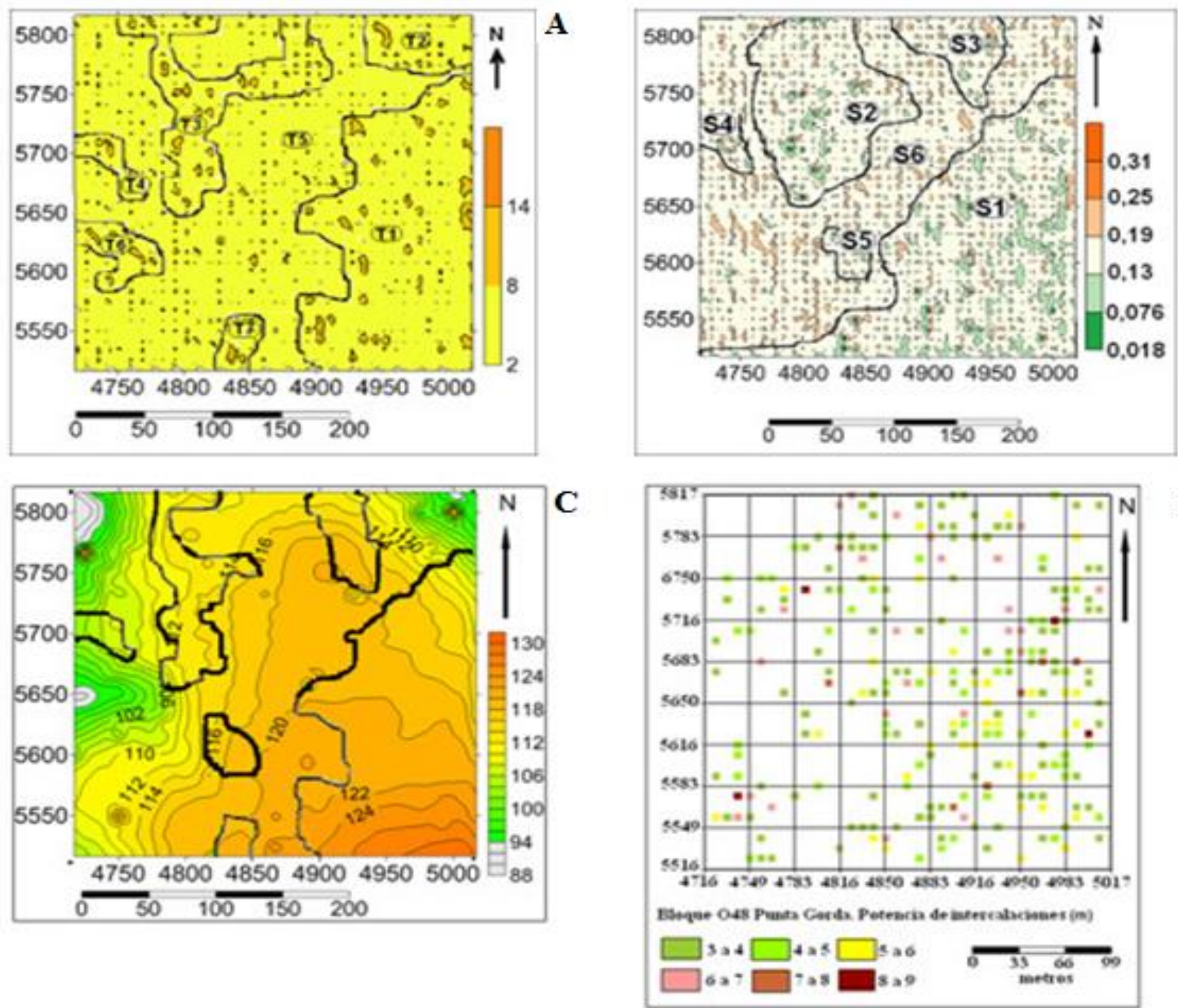

D

Figura 8. Distribución espacial de los indicadores de las intercalaciones en el bloque 048 de Punta Gorda. A) Índice de intercalaciones $I^{\tau}$ (\%). B) Entropía de las intercalaciones $\varepsilon_{I A}^{\tau}$. C) Cota de la superficie en metros sobre el nivel del mar. D) Potencia de intercalaciones en metros. 


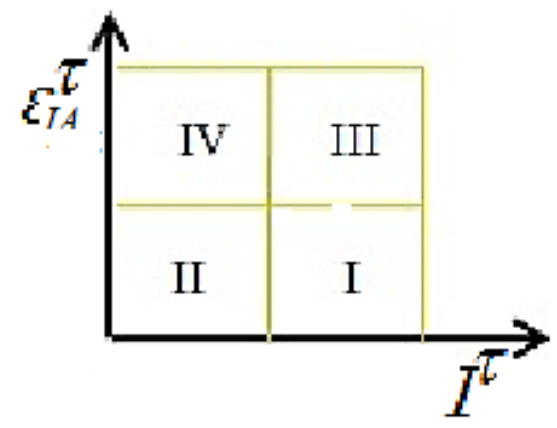

Figura 9. Condiciones para determinar las perspectivas de reducción de la dilución interna.

Del análisis realizado se teoriza que existen mayores probabilidades de reducción de la dilución interna en las áreas coincidentes donde se superponen los sectores $\mathrm{S} 1$ con $\mathrm{T} 1$, S3 con T2, S2 con T3 y S4 con T4. Las menores perspectivas de reducción de la dilución interna se obtienen de la combinación de S6 con T5, que es la franja orientada aproximadamente en dirección sublatitudinal, donde coincide con poca presencia de las IA pero de mayor dispersión. Se relacionan con IA de pequeñas dimensiones y muy separadas espacialmente, con poca influencia sobre la DI, al tiempo que se hace muy difícil su separación selectiva sin afectar las menas.

En la Figura 8 A se observa que en T6 $\mathrm{I}^{\tau}$ pronostica la presencia de IA donde la red de explotación no las detectó. En la estimación del bloque 048 se considera que $\varepsilon_{\mathrm{IA}}^{\top}$ es muy baja en el $78,3 \%$ y es baja en el $21,7 \%$ de los puntos; este comportamiento es similar al que poseen los cuatro yacimientos analizados. El total de los valores bajos y muy bajos se asocian a $\mathrm{I}^{\top}$ con probabilidades inferiores $\mathrm{o}$ iguales al $17 \%$, lo cual significa que las IA detectadas en el bloque presentan ese valor como máximo de probabilidad en el área del bloque; el valor de estimación más frecuente es el $6 \%$. Esta información influye en la estrategia para la reducción DI.

\section{CONCLUSIONES}

- Se demuestra que el modelo markoviano de yacimientos permite determinar la distribución probabilística espacial de las intercalaciones en yacimientos lateríticos cubanos.

- El índice $I^{\tau}$ proporciona la probabilidad de aparición de las intercalaciones y su entropía $\varepsilon_{I A}^{\tau}$ describe el grado de dispersión de estas. Ambos están definidos para cada dirección del yacimiento 
tomando cualquier punto de este como referencia y permiten representar la complejidad de la distribución espacial de las IA.

- En el bloque 048 del yacimiento Punta Gorda estos índices muestran las mejores condiciones para la extracción selectiva de IA en las áreas T1-S1, T2-S3 y T3-S2 y T4-S4, donde la superposición de los mapas de isovalores del índice $I^{\tau}$ y su entropía $\varepsilon_{I A}^{\tau}$ aportan la información necesaria para llegar a esta conclusión.

- El índice $I^{\tau}$ permite determinar que las IA en el bloque 048 se confirman en una extensión menor del $17 \%$ y como promedio en el 6 $\%$, ello muestra las reducidas dimensiones de las IA, lo que fija el nivel de selectividad minera para la reducción de la dilución interna.

\section{REFERENCIAS}

Cuador-Gil, J. Q. 2002: Estudios de estimación y simulación geoestadística para la caracterización de parámetros geólogoindustriales en el yacimiento laterítico Punta Gorda. Tesis doctoral. Universidad de Pinar del Río.

Duarte, M. C. 2009: Inferencia Estadística para Cadenas de Markov. Tesis de maestría. Universidad de Sonora. Hermosillo, México.

Durán, A. y Fernández, L. 2009: Aplicación de métodos de estadística multivariada y técnicas de clasificación en los minerales del Sector 10 del yacimiento Yagrumaje Oeste. En: III Convención Cubana de Ciencias de la Tierra. MIN2-06. La Habana, Cuba. ISBN: 978959-7117-19-3.

Fernández, L. F.; Rojas-Purón, A. y Belete, O. 2015: Parámetros y factores asociados a la Dilución Interna en yacimientos cubanos de níquel y cobalto. Minería y Geología, 31(4): 121-139.

Hernández, M. E. C.; Cobo, G. B. y Pérez, L. A. P. 2010: Aplicación del análisis de clúster a la clasificación de variedades de tabaco. INFOCIENCIA, 7(1).

Lavaut, W. 1987: Control litológico-mineralógico de la mineralización en la corteza de intemperismo de ultramafitas del campo mineral yacimientos Punta Gorda, Camariocas y Piloto. Tesis doctoral. Archivo E. G. S. Moscú.

Lavaut, W. 2004: Patrones de meteorización de rocas ofiolíticas de cuba oriental: su importancia para la minería. Minería y Geología, 20(3-4): 3-14. 
Legrá, A. A. 1999: Metodología para el pronóstico, planificación y control integral de la minería en yacimientos lateríticos. Tesis doctoral. Instituto Superior Minero Metalúrgico de Moa.

Martínez-Vargas, A. 2007: Estimación del error de geometrización empleando la geoestadística transitiva. Minería y Geología, 23(2): 14.

Peña-Abreu, R. E. 2015: Modelación matemática para optimizar el diseño de las redes del muestreo de exploración y explotación en yacimientos lateríticos de $\mathrm{Ni}$ y Co. Tesis doctoral. Instituto Superior Minero Metalúrgico de Moa.

Peña-Abreu, R. E. y Perdomo, E. A. 2014: Comportamiento probabilístico del hierro y el níquel en tres yacimientos lateríticos cubanos. Ciencia de la Tierra y el Espacio, 15(2): 161-171.

Peña-Abreu, R. E. y Vera-Sardiñas, L. O. 2013: Identificación de clases patrones para la modelación matemática en yacimientos de cubanos níquel. Ciencias de la Tierra y Espacio, 14(2): 154-169. Consultado: 20/12/2016. Disponible en:

http://www.redciencia.cu/geobiblio/paper/2013 Pena\%20y\%20V era Modelacion\%20matematica.pdf.

Peña-Abreu, R. E.; Matos, L.; Pérez, O.; Ortiz, E. y Robles, V. 2007: Propuesta de clases patrones en yacimientos lateríticos ferroniquelíferos. En: II Convención Cubana de Ciencias de la Tierra. La Habana, Cuba, 20-23 de marzo.

Tomás, J. 2017: Caracterización del comportamiento probabilístico espacial de la geoquímica en varios yacimientos cubanos de níquel. Tesis de maestría. Instituto Superior Minero Metalúrgico de Moa.

Vera-Sardiñas, L. O. 2001: Procedimiento para la determinación de las redes racionales de exploración de los yacimientos lateríticos de níquel y cobalto en la región de Moa. Tesis doctoral. Instituto Superior Minero Metalúrgico de Moa. 